\title{
Correction to: Diagnostic efficacy of cone beam computed tomography in paediatric dentistry: a systematic review
}

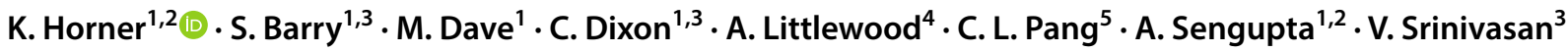

Published online: 3 February 2020

(c) European Academy of Paediatric Dentistry 2020

\section{Correction to: European Archives of Paediatric Dentistry https://doi.org/10.1007/s40368-019-00504-x}

In the original publication of the article the fifth author's name "A. Littlewood" was submitted as "A. Littewood" which was left unnoticed in the later stages. The correct name is as published in this erratum.

Publisher's Note Springer Nature remains neutral with regard to jurisdictional claims in published maps and institutional affiliations.

The original article can be found online at https://doi.org/10.1007/ s40368-019-00504-x.

\section{K. Horner}

keith.horner@manchester.ac.uk

1 Division of Dentistry, School of Medical Sciences, Faculty of Biology, Medicine and Health, University of Manchester, Manchester Academic Health Science Centre, Coupland Building 3, Manchester M13 9PL, UK

2 Dental Radiology, University Dental Hospital of Manchester, Manchester University NHS Foundation Trust, Manchester Academic Health Science Centre, Higher Cambridge Street, Manchester M15 6FH, UK

3 Paediatric Dentistry, University Dental Hospital of Manchester, Manchester University NHS Foundation
Trust, Manchester Academic Health Science Centre, Higher Cambridge Street, Manchester M15 6FH, UK

4 Information Specialist, Cochrane Oral Health, Division of Dentistry, School of Medical Sciences, Faculty of Biology, Medicine and Health, University of Manchester, Manchester Academic Health Science Centre, Coupland Building 3, Manchester M13 9PL, UK

5 Division of Imaging, Manchester Royal Infirmary, Manchester University NHS Foundation Trust, Manchester Academic Health Science Centre, Oxford Road, Manchester M13 9WL, UK 